\title{
Sia: The Sugar Code of Life
}

\author{
Robert Skopec* \\ Researcher analyst, Slovakia \\ *Corresponding author: Robert Skopec, Researcher analyst

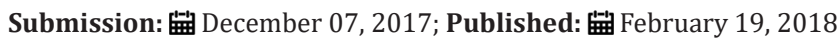

\begin{abstract}
Our cells are coated with sugar, and when it comes to cancer, that's anything but sweet. In a recent talk at TED x Stanford, chemist Carolyn Bertozzi explained why. She studies sialic acid, a sugar that seems to deceive the immune system, allowing cancer cells to evade the body's defenses. This work focuses on the complex, sugary structures surrounding human cells. That foliage-like coating, it turns out, can tell us a lot of our body - it even reveals a patient's blood type. Sugar and carbohydrates are dangerous supporters of different types of cancer.
\end{abstract}

\section{Introduction}

As it can be seen from the information of the American Chemical Society (ACS), ideally, the immune system can figure out which cells are bad, attack them and protect the body from disease. In the case of cancer cells, though, a special sugary coating tricks the immune system into ignoring them. The sialic acid, a sugar that's denser in cancer cells than in other cells. It seems deceive the immune system, allowing the cancer cells to evade the body's defenses. Unnoticed and unchallenged, cancer cells are free to divide and run wild inside the body $[1,2]$.

\section{Monosaccharides - Sialic acid/N - acetylneuraminic acid}

$\mathrm{N}$-acetylneuraminic acid, also known as Sialic acid, is a key component of important amino sugars that mediate cellular communication. Sialic acid is a naturally-occurring building block for compounds such as glycoproteins and gangliosides, and is therefore found in cell membranes, in the plasma and in glandular secretions such as saliva (Figure 1).

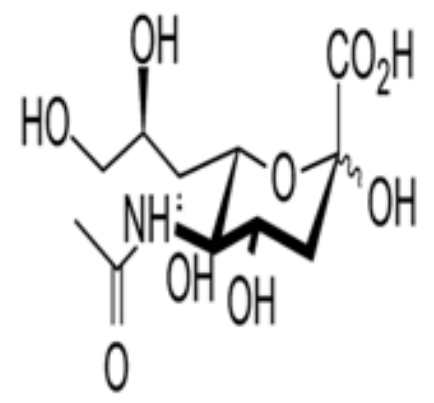

Figure 1: Jennewein Biotechnologie GmbH 2017.

Due to the high concentration of Sialic acid in gangliosides, which are found in the membranes of neurons, this monosaccharide has an important role in brain development, and in the function of the nervous system. In addition, several viral pathogens (including human influenza virus and avian influenza virus) use sialylated proteins as receptors.

\section{Key change of malignant tissue glycosylation is altered sialic acid processing}

During malignant transformation, glycosylation is heavily altered compared with healthy tissue due to differential expression of glycosyltransferases, glycosidases and monosacharide transporters within the cancer environment. One key change of malignant tissue glycosylation is the alteration of sialic acid processing that leads to a general upregulation of sialylated glycans (hypersialylation) on cell surfaces and an increased introduction of the non-human sialic acid N-glycolyl-neuraminic acid (Neu5Gc) instead of $\mathrm{N}$-acetyl-neuraminic acid into cell surface glycans $[3,4]$.

These changes have been shown to be the result of altered sialyltransferase and sialydase expression. Functionally, cancer associated hypersialylation appears to directly impact tumor cell interaction with the microenvironment, in particular the modulation of sialic acid-binding lectins on immune cells. Moreover, Neu5Gc expression in human tissues enhances inflammation due to an anti-Neu5Gc immune response, which can potentially influence inflammation-induced cancer and cancer-associated inflammation [5].

Changes in glycosylation are a classic hallmark of malignant transformation. The mechanisms that produce these aberrant glycosylation patterns are broad, because glycosylation is not template driven, but dependent on multiple interactions resulting from gene expression, substrate availability, the cellular environment and the underlying protein structure. Glycans are attached to both proteins and lipids to make glycoproteins and glycolipids respectively. Classical types of glycosylation are $\mathrm{N}$ - and O-linked (on proteins), glycosphunolipids (on cell- membrane sphingosine), glycosaminoglycans (GAGs) (protein-bund and 
free) and glycophosphatidylinositol anchors (plasma-membrane glycolipid and a protein attached through glycans) [6].

The type of glycosylation that is finally presented at a given glycosylation site is heterogenous, giving rise in some cases to many glycoforms. During organism development, the differentially expressed glycoforms cue signalling for tissue modeling in a rapid and dynamic manner. In some ways, the unusual glycosylation seen in cancer is adapted from roles in development. For example, the early discovered antigenic structures found on tumor cell were glycans that had already been described in foetal development.

$\mathrm{N}$-Glycans on tumor cells are often increasingly branched. This has been explained by upregulation of the enzyme GlcNAcT-V, which adds a second GlcNAc monomer to the core pentasaccharide strucure producing dominantly tri-antennary glycan structures that enhance metastasis in animal models. Malignant transformation of epithelial cells is associated with secretion of both membrane and secreted O-glycans, which often carry altered glycosylation patterns systematically. These cancer mucins can be detected in blood, and have prognostic value. Classic examples include episialin (MUC1) expressed by many carcinomas including breast and ovarian, and CA125 (MUC16), which is overexpressed by ovarian cancer cells and is used in clinical routine. Beside mediating interactions with sialic acid-binding receptors, mucins have a high negative charge associated with their structure that inhibits adhesion of tumor cells within the tissue matrix, and advances metastasis.

They may also provide a physical block for blood-borne tumor cells against immune cell interaction. O-linked $\mathrm{Tn}$ and $\mathrm{T}$ antigens are produced by incomplete glycosylation of mucins. These structures occur infrequently in adult organisms and as such are targets for an adaptive immune response that leads to the generation of antibodies. These glycan-targeted antibodies have potential prognostic value, and studies are underway to investigate enhancing the immune response against thes tumor-specific ligands [7].

Regardless of the change in the underlying glycan structure, the upregulation and alteration of terminal sialic acid structures (Sia) is a hallmark of cancer. Classically, this results in reduced adhesion of the tumor cells to the extracellular matrix allowing in some cases for an increased plasticity of the tumor cells within the tissue matrix, and may also serve to mask detection by the alternative pathway of complement activation. The upregulation of sialylation has been identified to have another role as ligands for Sia-binding lectins including immune cell expressed Siglec receptors and selectins. The known alteration in Sia structure and enzymatic processing in cancer lead to recent advances in our understanding of the relationship between cel, surface sialylation in cancer progression, concluding with the clinical prospects for targeting Sia-based immune cell interactions as a potential patway to boost anti-tumor immunity [8].

\section{Sialic acid changes and their role in tumorigenesis and cancer progression}

Intracellular changes in sialyltransferase expression alters glycan processing within the Golgi. Changes in sialidase expression effects the sialic acid status of glycans during the processing, transport in lysosomes, and the cell membrane. Protein bound Neu5Gc from dietary sources is metabolically processed and incorporated into endogenous glycans, which is in combination with an anti-Neu5Gc immune response generates xenosialitis, a source of cancer-associated inflammation [9].

\section{Cancer-associated Sia modifications}

The Sia family of sugars share a characteristic 9-carbon chain backbone with a carboxylic acid residue attached to carbo-2, the anomeric center. The types of mammalian Sia, include the known cancer antigens. Sia strucure differs from the hexoses in having only two hydroxyl residues attached directly to the hexose ring. The amine at position C-5 is modified predominantely as N-acetyl, and N-glycol, but can also be a hydroxyl or amine. The aliphatic side-chain of three hydroxyl residues at C- 6 can be modifiesd with acetyl, methyl, sulfate, or phosphate residues. The 4-OH can also be acetylated, however, very little is known about function of this group.

The structural flexibility innate within the Sia backbone increases the potential chemical information that can be stored within these molecules when compared with the hexoses and pentoses. The amount of information in the form of chemical complexity that each glycan monomer contains has led to them being described as a sugar code. Sias are therefore potentially the most versatile of the glycan platforms $[10,11]$.

Sialic acid and its derivatives, and sialylated compounds (i.e. molecules conjugated to sialic acid) are already used as active pharmaceutical ingredients to prevent viral infections. Additional research will focus on the use of these compounds to promote healthy brain development and to prevent age-related degenerative nervous system disorders [11]. The sialic acid that we produce is a crystalline product with high purity [12].

The news isn't all bad. Researchers plan to use drugs to strip the sugars away from cancer cells. Once these cells' secret is exposed, the immune system may be able to devour them before they have a chance to take hold. It includes what else our cells' coating might be able to do - and how these revelations might change the way we fight cancer [13].

Over four decades ago, specific tumor characteristics were ascribed to the increased expression of sialic acid sugars on the surface of cancer cells, and this led to the definition of sialic acids as potential therapeutic targets. Recent advances in glycobiology and cancer research have defined the key processes underlying aberrant expression of sialic acids in cancer, and its consequences, more precisely. These consequences include effects on tumor growth, escape from apoptosis, metastasis formation, and resistance to therapy. Collectively, these novel insights provide further rationale for the design and development of therapeutic approaches that interfere with excessively high expression of sialic acids in cancer cells (Figure 2). Strategies to target aberrant sialylation in cancer, however, have evolved comparatively slowly. Here, we review recent 
findings that emphasize the detrimental effects of hypersialylation on multiple aspects of tumor growth and behavior. We also discuss novel therapeutic strategies [14].

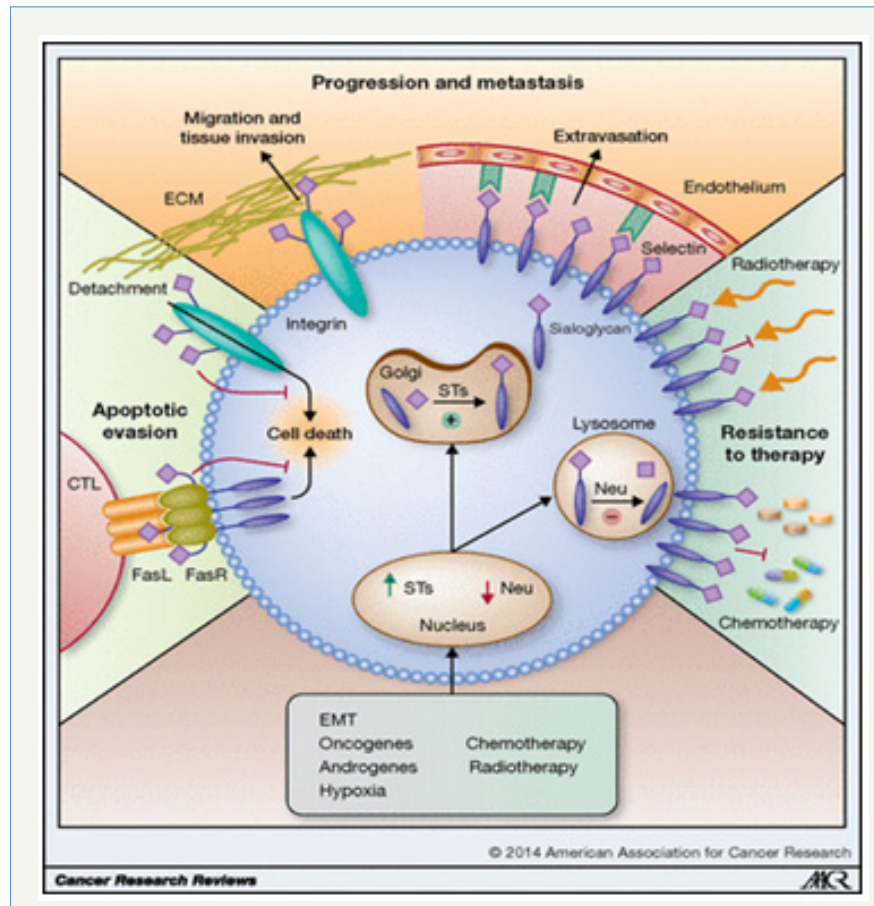

Figure 2: 2014 American Association for Cancer Research

Aberrant sialylation favors tumor growth and progression Several factors (e.g., oncogenes, hormones, or chemotherapy) increase the expression of sialyltransferases and downregulate the expression of sialidases (Neu) in cancer cells (red). As a result, sialoglycan synthesis in the Golgi system by sialyltransferases is enhanced and hydrolysis of sialoglycans by sialidases in the lysosome is reduced, leading to accumulation of hypersialylated structures on the cell membrane. The aberrant high expression of sialoglycans impairs apoptotic signaling by the Fas receptor or integrins following detachment from the ECM (green); facilitates binding to the ECM or selectins, allowing migration/tissue invasion and metastasis formation, respectively (orange); and mediates resistance to chemo- and radiotherapy (blue). EMT, ephithelialmesenchymal transition. (C2014 American Association for Cancer Research)

\section{The immune response like the cancer can evolve}

Fact, that infections could cause a cancer retreat, or even vanish, was known well before the advent of modern medicine. In early modern Europe doctors used septic dressings on tumors with ulcers, and deliberately created purulent sores. By the end of the $19^{\text {th }}$ century, Wiliam Coley, a bone surgeon in New York, was methodically infecting patients with Streptococus bacteria.

Coley's work fell out of favor, partly to the rise of radiation therapy. Many others continued to cling to the idea that the immune system might in some circumstances be provoked into recognizing, attacking and subduing a cancer. In 1976 this latent belief in the potential of "immunotherapy" blossomed into hope with the discovery of interleukin 2 (IL-2). It is a growth factor that encourages the production of T-cells, white blood cells that scan the body for unwanted invaders and, on finding them, activate other parts of the immune system, including the B-cells which produce antibodies.

But IL-2 was a false dawn. On its own, it activated the immune system indiscriminately, and as it said Elad Sharon, at the National Cancer Institute, the immune system is a powerful beast. Answers started to arrive in the 1990s. James Allison, at the Cancer Research Laboratory at the University of California, Berkeley, began work on a protein called CTLA- 4 on the surface of some T-cells. By 1996 he had shown that this protein put a brake on the immune response to cancer. Blocking CTLA-4 with an antibody removed the brake, the immune system activated itself and go to work. Tumors in mice vanished when the animals were given CTLA-4 blocking antibodies [15].

At the time oncologists were unimpressed by Dr. Allison's results. Cancer had been cured in mice many times over. After many failed trials, immunotherapy was in exile - banished to the small corners of the big oncology meetings. But in 1999 Tasuko Honjo of the University of Kyoto, Japan, showed that the gene for a protein called PD-1 also seemed to tamp down the immune system. When this gene was switched off in mice, some developed autoimmune diseases - a sign of an immune system in overdrive. In collaboration with Arlene Sharpe and Gordon Freeman at Harvard, Dr. Honjo showed that some cancer cells had a second protein called PD-L1 on their surfaces which, by interacting with the PD-1 on T-cells protected the cancer from them.

Then, in 2010, Bristol-Myers Squibb released results from a trial of an anti-CTLA-4 antibody, Yervoy (imilimumab), in malignant melanoma. Compared with the state of the art, they were fantastic. It was the first drug shown to change survival in this devastating disease, raising the median to ten months. Some survived much longer.

Because the immune system is such a powerful beast, evolution has equipped it with a system of checks and balances [11] Both CTLA-4 and PD-1 are parts of that system. When one sort of immune cell presents an antigen which it has picked up to another, the second cell will ignore it if, at the same time, the first cell stimulates the CTLA- 4 receptor. If the CTLA- 4 receptor is blocked with an antibody like Yervoy, though this checkpoint system does not work. Unchecked, the immune system is able to react to a wider range of antigens - including tumor antigens. Freed up by Yervoy the body's T-cells started attacking the melanomas. It turne out, kept on attacking them. The most remarkable feature of the new checkpoint inhibitor was that small subset of patients survided for year after year.

Despite indications of success with melanomas, many scientists thought the checkpoint inhibitor mechanism would not be broadly effective. Melanomas accumulate a very large array of mutations, and are thus more likely than most cancers to display antigens which trigger an immune response. Pessimists have a pretty good 
record when it comes to cancer prognostication. But this time they were wrong. At Merck Roger Perlmutter, an immunologist became interested in PD-1 blocker then known as MK-3475. Unlike CTLA4, which works higher up the immune system, PD-1 has a frontline role; if a cancer cell carries PD-1's counterpart, PD-L1, on its surface, T-cells will ignore the cell despite any suspicious antigens it may be carrying. MK-3475 seemed to block the interaction nicely [16].

The expansion was in part a response to a new discovery: early evidence suggested that checkpoint inhibitors could also results with lung cancers. Luis Diaz, head of solid-tumor oncology at the Memorial Sloan Kettering Cancer Centre in New York, recalls that was completely unexpected. It was a success of the believers in immunotherapy. Jedd Wolchok, a medial oncologist at Memorial Sloan Kettering, says immunotherapies do not have the same kinds of impact as other types of cancer therapy. It looks like a chronic struggle between a patient's immune system and cancer [11]. This apparent equilibrium is quite different from what is seen in chemotherapy, where the cancer will be either susceptible or resistant. The difference seems to be due at least in part to the fact that the immune response, like the cancer, can evolve [11]

Improving immune response is perhaps today's biggest oncological challenge - the source of more excitement, and investment, than any other recent development in the field. Part of the answer will come from a better understanding of the steps needed to generate an anti-tumor response from the immune system, and the therapeutic targets available. Herve Hoppenot, the boss of biotech firm Incyte, based in Wilmington, Delaware, says that some tumors protect themselves from the immune system using another checkpoint, ID01 (an enzyme that was first discovered in a search for ways to protect a fetus from immune rejection). Incyte is testing epacadostat, an existing drug known to inhibit ID01, as a cancer treatment both alone and in combination with PD-1 blockers. Dr. Freeman at Harvard says he has been told there are over 80 Chinese groups developing different PD-1 antibodies.

\section{The Best Diet According to Harvard Researchers}

If you want to lose weight, what's on your plate is often more important than the minutes you spend in the gym. And if you want to see the most change, a study from Harvard says that you should be cutting carbohydrates (carb), not only fat.

For the study, published in journal PloS One, researchers from Harvard and Brigham and Women's Hospital reviewed 53 randomized trials of over 68,000 patients who had been assigned to either low-fat or low-carb diets. They found that low-carb diets were consistently better at helping patients lose weight than low-fat diets. The participants on the low-carb diets lost 2.5 pounds more than those on low-fat diets, with an average weight loss among all groups at about 6 pounds.

Another study on the weight-loss benefits of a low - carb diet adds further evidence that if you want to lose weight, ditching bread - not olive oil - can help you see success. Another recent study also showed that dieters who ate fewer than 40 grams of carbohydrates per day lost about 8 pounds more than dieters who were put on a low-fat diet. Several other studies have shown that high - carb diets may be the real heart-disease culprit, not only saturated fat [2].

All in all, this new review is a good reminder that if you want to lose weight, you should choose a diet in healthy fats, lean proteins, and fresh produce. Of course, not all fats are created equal - you must find out which healthy fats are recommended by science to be incorporating into your diet [17].

\section{The unusual method of cooking rice traces the poison arsenic}

Experiments suggest normal method for cooking rice can expose consumers to range of health problems including heart disease, diabetes and cancer. Millions of people could be putting themselves at risk by cooking their rice incorrectly, scientists have warned. Recent experiments show a common method of cooking rice - simply boiling it in a pan until the water has steamed out can be insufficient in preventing exposure to traces of the poison arsenic, which contaminates rice while it is growing as a result of industrial toxins and pesticides.

The chemical has been linked to a range of health problems including heart disease, diabetes and cancer, as well as development problems. While it is generally believed traces of arsenic are expelled when the rice is cooked, it has now been claimed this only takes place adequately when the rice is soaked overnight.

Andy Meharg, professor of biological sciences at Queens University Belfast, tested three ways of cooking rice for the BBC programme 'Trust Me, I'm a Doctor', to see whether it altered the levels of arsenic. In the first method, Professor Meharg used a ratio of two parts water to one part rice, where the water was "steamed out" during cooking - a method commonly used. He found this left most of the arsenic present.

By contrast, when the he used five parts water to one part rice and washed the excess water off, levels of arsenic were almost halved, while in the third method, in which the rice was soaked overnight, levels of the toxin were reduced by 80 per cent.

The safest method of cooking rice is therefore to soak it overnight, then wash and rinse it until the water is clear, before draining it well and boiling in a saucepan, with a ratio of five parts water to one part rice.

According to 2014 research from Channel 4's Dispatches and The Institute for Global Food Security, around 58 per cent of ricebased products in the UK contained high levels of arsenic. However, new legal limits were introduced last year by the EU in response to safety concerns $[2,18]$.

\section{Without sex you get stronger immune system}

Researchers found that mealworm beetles who avoid mating tend to live longer. It's thought sex weakens their immune system - leaving them vulnerable to bugs, Despite the findings being on insects, they believe it could apply to humans. Experts believe not 
having sex will help people to live longer - explaining why nuns tend to outlive most.

Desperate for a magical potion to help them live longer, many frantically try a range of new things in the belief it will give them extra 10 years of life. But now scientists believe they've found out exactly how you can extend your lifespan - but it won't be an easy change for many. Refraining from having sex will help you live longer than all of your peers, a study and experts believe it could explain why nuns - who stay away from the act completely - tend to live longer [19].

Researchers from the University of Sheffield discovered that mealworm beetles live longer if they avoid mating. While those who tried to produce offspring each day passed away at a much younger age. Mating was found to release a hormone in the insects needed to produce sperm in a male or eggs in a female.

But this was also found to have a negative effect on their immune system, the researcher found. This makes them vulnerable to potentially deadly infections as their body's natural ability to fight rogue bacteria is compromised. Despite the mechanism not being the same in humans, study author Dr. Michael Siva - Jothey believes the principle can be applied.

Nuns tend to have a longer lifespan than women with children and most people know of someone with a maiden aunt who seems to live forever. The question is, why? The beetles which mate die sooner than beetles which don't mate. Humans also have weaker immune system during sex, which explains how STIs can be easily spread. Having sex was found to have a negative effect in the immune system of mealworm beetles - making them vulnerable to infections [20].

Nuns, priests, vicars and monks are known to live longer than others - but not of their religious protection. It is believed their low rates of high blood pressure - which increases the risk of heart disease and stroke - are responsible. This comes after research revealed that people who eat hot chilli peppers were 13 per cent less likely to die early. Vermont University researchers found that capsaicin - responsible for the fiery kick - seemed to ward off heart conditions and stroke. While experts from Massachussetts General Hospital found going vegan could add years to someone's life.

Eating less protein from animal sources and instead swapping it for cereals, beans and soya substantially reduced death rates, they discovered. Mortality from heart diseases fell 12 per cent, and deaths from all causes - including cancer - were reduced by 10 per cent [21].

\section{Sia: The Sugar Code of Life}

Recent studies on sialic acid (Sia - a sugar) shine a bright light on an established risk factor for cancer. Scientists and doctors have known long-term consumption of red meat (beef, pork and lamb) is highly correlated to cancer [2]. The researchers centered their efforts on a non-human form of sialic acid (Neu5Gc) that is present in significant quantities in red meat but not poultry or fish (except caviar). The human version of this sugar sialic acid is Neu5Ac or
$\mathrm{N}$-acetylneuraminic acid. Researchers were able to demonstrate that while the non-human glycan is not produced by humans it does show up on human epithelial cell surface sugar structures (glycoproteins). Epithelial cells line our throat and intestinal tract. It is especially common in cancerous tissue [22].

The only way this non-human sialic acid could be embedded on human glycoproteins (sugar antennas) is through diet. Red meat contains this non-human sialic acid sugar. That is the source. If we eat a lot of red meat, the glycoproteins on our cells can have this non-human form of sialic acid incorporated within them. The non-human sialic acid is what causes inflammation and is linked to cancer. The human form of sialic acid does not cause this type of problem. It is anti-inflammatory and supports the immune system. Since epithelial (surface) cells are replaced every few days, eating red meat sparingly gives the body a chance to replace this non-human form of sialic acid accumulated from the diet with the human form. This gives the body a chance to rest from inflammation and disease creation. A constant diet of red meat gives the body no time to heal.

Metabolic incorporation (glycosylation) of dietary Neu5Gc into human tissue makes this glycan the first example of a xenoautoantigen which can react with circulating anti-Neu5Gc antibodies (xeno-antibodies). The resulting antigen-antibody interaction is hypothesized to promote chronic inflammation or 'xenosialitis' which would contribute to carcinogenesis or to other diseases exacerbated by chronic inflammation. it appears that glycosidically bound Neu5Gc is the dietary source that is bioavailable for tissue incorporation and not the free monosacharide $[23,24]$.

This research explains why eating too much red meat is linked to cancer. Many healthy diets either eliminate red meat or allow it only sparingly. The wisdom of this advice has just been verified and validated by the field of glycobiology-the science that studies sugar!

Vegetables, fruit, fish, shellfish, chicken, turkey, eggs, butter do not contain the non-human sialic acid. Caviar, beef, goat's milk cheese, pork, bison, cow's milk cheese, and lamb (in descending order of amount) contain the non-human version of sialic acid. These foods should be minimized [2]. The science of nutritional glycobiology continues to evolve. The actual question is: why disease occurs locked in the sugar code of life [2].

\section{References}

1. Schauer R (2000) Achievements and challenges of sialic acid research. Glycoconj J 17(7-9): 485-499.

2. Skopec RI (2017) Coding By Quantum Entanglement Entropy. American Research Journal of Biosciences 2(3): 1-7.

3. Severi E, Hood DW, Thomas GH (2007) Sialic acid utilization by bacterial pathogens. Microbiology 153(Pt 9): 2817-2822.

4. Severi E, Müller A, Potts JR, Leech A, Williamson D, et al. (2008) Sialic acid mutarotation is catalyzed by the Escherichia coli beta-propeller protein YjhT. J Biol Chem. 283(8): 4841-4891.

5. Fulcher CA (2009) MetaCyc Chimeric Pathway: superpathway of sialic acid and CMP-sialic acid biosynthesis.

6. Traving C, Schauer R (1998) Structure, function and metabolism of sialic 
acids. Cellular and Molecular Life Sciences CMLS 54(12): 1330-1349.

7. Skopec R (2017) De-Programming Colorectal Cancer by Increase in Cholesterol Level. International Journal of Cancer Research \& Therapy 2(1): 1-4.

8. Warren, Leonard, Felsenfeld, Herbert (1962) The Biosynthesis of Sialic Acids. The Journal of Biological Chemistry 237(5): 1421-1430.

9. Hai Yu, Harshal Chokhawala, Shengshu Huang, Xi Chen (2006) One-pot three-enzyme chemoenzymatic approach to the synthesis of sialosides containing natural and non-natural functionalities. Nat Protoc 1(5): 2485-2492.

10. Pearce OMT, Läubli H (2016) Sialic acids in cancer biology and immunity Glycobiology 26(2): 111-128.

11. Skopec R (2017) Agriculture As Producer of World Cancer Epidemic MOJ Biology and Medicine 1(6): 00034.

12. Fuster, Mark M, Esko, Jeffrey D (2005) The sweet and sour of cancer: Glycans as novel therapeutic targets. Nature Reviews Cancer 5(7): 526542.

13. https://www.washingtonpost.com/national/health-science

14. Bull C, Stoel MA, den Brok MH, Adema GJ (2014) Sialic acids sweeten a tumor's life. Cancer Res 74(12): 3199-3204.

15. Varki A, Gagneux P (2012) Multifarious roles of sialic acids in immunity. Ann N Y Acad Sci 1253(1): 16-36.

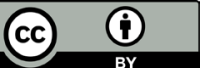

Creative Commons Attribution 4.0

International License

For possible submissions Click Here
16. Jürgen Sandow, Ekkehard Scheiffele, Michael Haring, Günter Neef, Klaus Prezewowsky, Ulrich Stache (2007) Hormones. Ullmann's Encyclopedia of Industrial Chemistry ( $7^{\text {th }}$ edn), Wiley, pp. 1-81.

17. Shy L: https://www.yahoo.com/lifestyle/diet-want-loose-weightaccording-103000664.html

18. BBC (2017) Scientists warn that the usual method of cooking rice. Traces the poison arsenic.

19. Wang B (2012) Molecular Mechanism Underlying Sialic Acid as an Essential Nutrient for Brain Development and Cognition. Adv Nutr 3(3): 465S-472S

20. Ponsot G (2007) Enfermedades por depósito de ácido siálico libre: enfermedad de Salla (incluida su forma infantil grave) y sialuria. EMCPediatría 42(1): 1-3.

21. Matthews S: http://www.dailymail.co.uk/health.article-4124096/ Want-live-longer-Avoid-having-sex-Nuns-stronger-immune-systemsvulnerable-infections.

22. Varki, Ajit, Roland Schauer (2008) In Essentials of Glycobiology. Cold Spring Harbor Press, USA. p. 14

23. Samraj A, Pearce OM, Läubli H, Crittenden AN, Bergfeld AK, et al. (2015) A Red Meat-derived Glycan Promotes Inflammation and Cancer Progression. PNAS 112(2): 542-547.

24. Varki A (2010) Colloquium paper: Uniquely human evolution of sialic acid genetics and biology. Proc Natl Acad Sci U S A 107(Suppl 2): 89298946

\section{Your subsequent submission with Crimson Publishers} will attain the below benefits

- High-level peer review and editorial services

- Freely accessible online immediately upon publication

- Authors retain the copyright to their work

- Licensing it under a Creative Commons license

- Visibility through different online platforms

- Global attainment for your research

- Article availability in different formats (Pdf, E-pub, Full Text)

- Endless customer service

- Reasonable Membership services

- Reprints availability upon request

- One step article tracking system 